\title{
Effect of Hydroalcoholic Extract of Kelussia odoratissima Mozaffarian on Uterus Contractions of Mature Rats
}

\author{
Jafarzadeh L. ${ }^{1} M D$, Habibian R. ${ }^{2} M D$, Rafieian Kopaei M.* PhD, Mohammadzadeh Z. ${ }^{3} M D$
}

\begin{abstract}
*Medical Plants Research Center, Shahrekord University of Medical Sciences, Sharekord, Iran 1 Obstetrics and Gynecology Department, Shahrekord University of Medical Science, Shahrekord, Iran ${ }^{2}$ Infectious Disease Department, Medicine Faculty, Shahrekord University of Medical Science, Shahrekord, Iran ${ }^{3}$ Medical Sciences Department, Faculty of Medicine, Shahrekord University of Medical Science, Shahrekord, Iran
\end{abstract}

\begin{abstract} plant has been mentioned in Iranian traditional medicine. The aim of study was contractions of adult rats. rats); receiver of Tyrod solution (first group) and receiver of Dejalon solution Oxytocin. The $0.125,0.25$ and $0.5 \mathrm{mg} / \mathrm{ml}$ final concentrations of the alcoholic statistically analyzed by SPSS 17 software using repeated measures ANOVA. concentration dependent manner. contractions of adult rat uterus in a concentration dependent manner.

\section{Keywords}

Kelussia odoratissima [Not in MeSH];

Uterine Contraction [http://www.ncbi.nlm.nih.gov/mesh/68014590];

Rats [http://www.ncbi.nlm.nih.gov/mesh/68051381];

Potassium Chloride [http://www.ncbi.nlm.nih.gov/mesh/68011189];

Oxytocin [http://www.ncbi.nlm.nih.gov/mesh/68010121]
\end{abstract}

Aims: Kelussia odoratissima is a plant from Umbelliferae family that has nutritional and pharmacological properties. The antispasmodic effect of this to assess the effect of alcoholic extract of Kelussia odoratissima on uterus

Materials \& Methods: In this experimental study, 16 adult female Wistar rats were studied and were randomly divided into two groups (each consisted of 8 (second group) groups. A $10-15 \mathrm{~mm}$ sample of uterus smooth muscle were placed in an organ bath containing Tyrod or Dejalon solution (10ml), and contractions were recorded with Isometric method after inducing with $\mathrm{KCl}$ and extract of Kelussia odoratissima was added to the organ bath. Data were

Findings: The alcoholic extract of Kelussia odoratissima significantly decreased the KCl-induced $(\mathrm{p}<0.001)$ and Oxytocin-induced $(\mathrm{p}<0.002)$ contractions in a

Conclusion: The alcoholic extract of Kelussia odoratissima inhibits the

\author{
*Corresponding Author \\ Tel: +983833346692 \\ Fax: +980833349506 \\ Address: Medical Plants Research Center, Shahrekord University of Medical Sciences, Rahmatiyeh, Shahrekord, Iran \\ rafieian@yahoo.com \\ Received: May 1, 2015




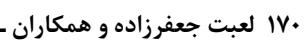

جلوگيرى از حاملكى است كه داراى عوارض جانبى متعدد هستند

مطالعات مختلف نشان داده است كه زنان تمايل زيادى به استفاده

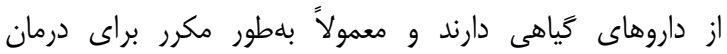

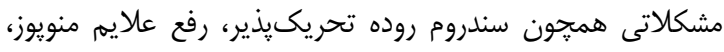

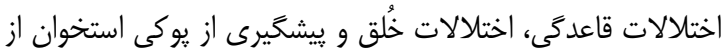

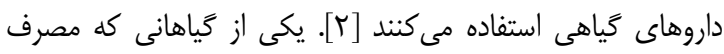

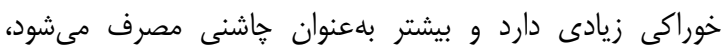

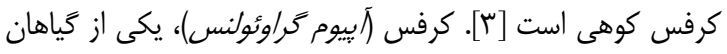

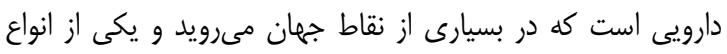

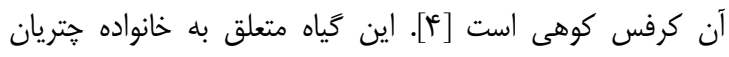

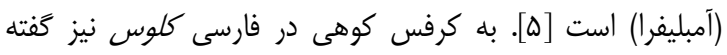

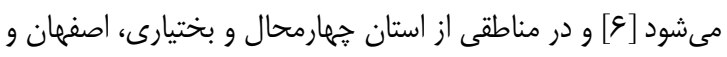

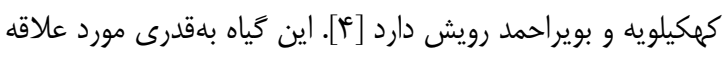

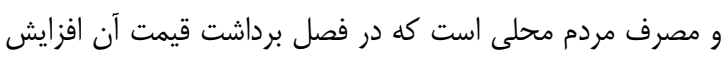

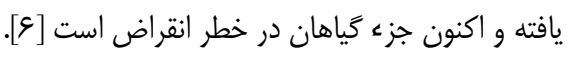

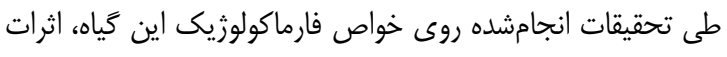

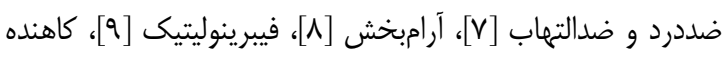

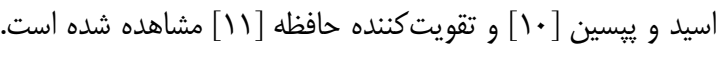
همجنين بر طبق مطالعات انجامشده، اين كياه داراى تركين

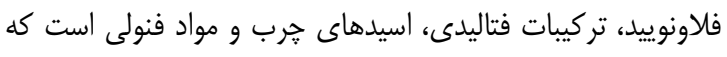

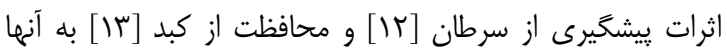

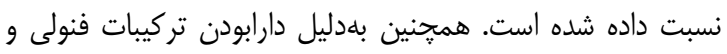

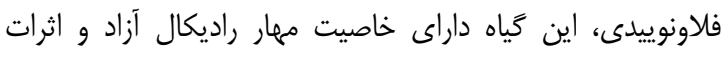

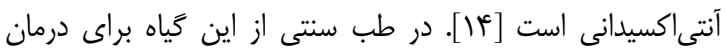

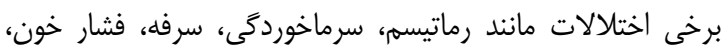

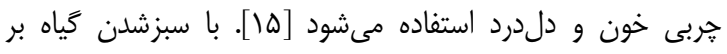

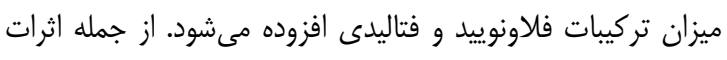

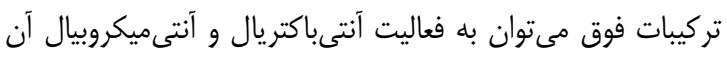

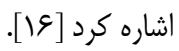

تحقيقات انجامشده حاكى از آن است كه اين كياه مىتواند بلهورو

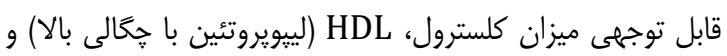
LDL

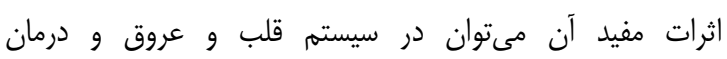

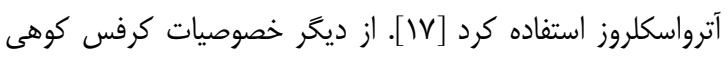

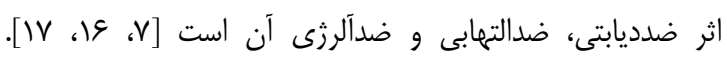

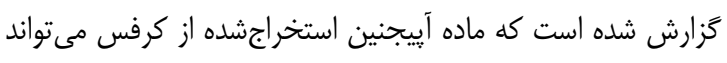

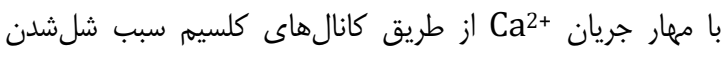

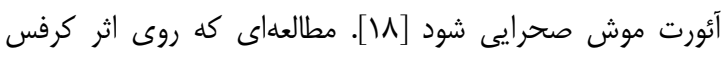

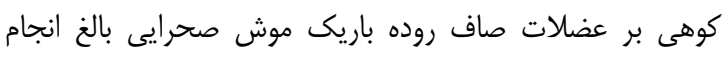

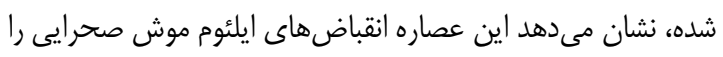

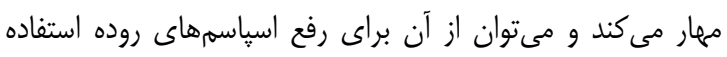

اثر عصاره الكلى كرفس كوهى بر انقباضات

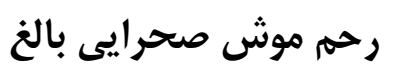

MD العبت جعفرز اده كروه زنان و زايمان، دانشكاه علوم يزشكى شهركرد، شهركرد، ايران

MD رويا حبيبيان

كروه بيمارىهاى عفونى، دانشكده يزشكى، دانشكاه علوم بزيكى شهركرد، شهركرد، ايران

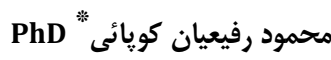

مركز تحقيقات كياهان دارويى، دانشخاه علوم يزشكى شهركر دمد، شهركرد، ايران

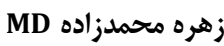

كروه علوم بزشكى، دانشكده بز شكى، دانشكاه علوم بزشكى شهر كرد، شهركرد،

ايران

جكيده

اهداف: كرفس كوهى، كياهى از خانواده جتريان و داراى خواص غذايى

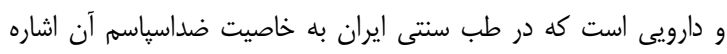

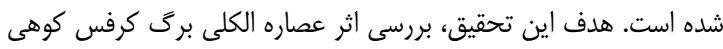

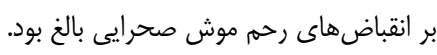

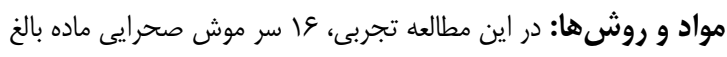

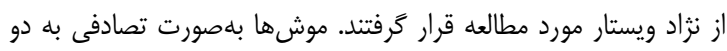

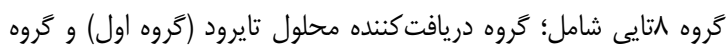

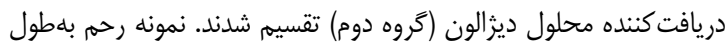

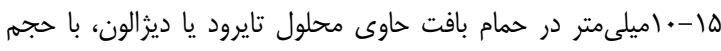

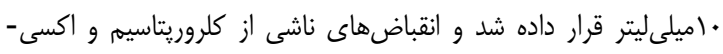

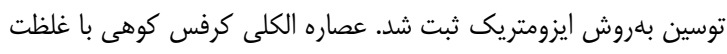

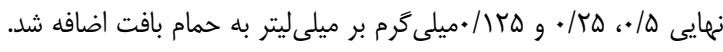

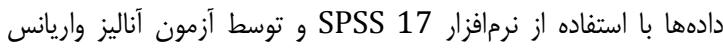

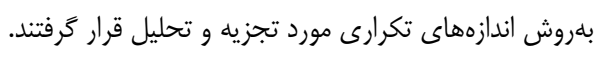

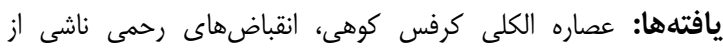

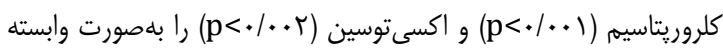

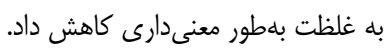
نتيجلَّيرى: عصاره الكلى كرفس كوهى، انقباض كادهاى رحم موش

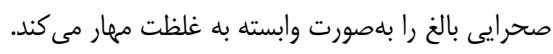
كليدوازهها: كرفس كوهى، انقباضات رحمى، موش صحرايى، كلروريتاسيه، اكسىتوسين

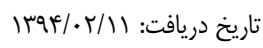

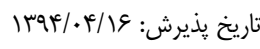
rafieian@yahoo.com :تويسنده مسئول:

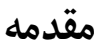

درمان متداول ديسمنوره يا انقباضهاى دردناك عضلات رحمه،

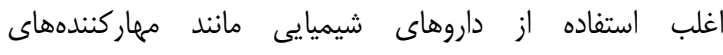

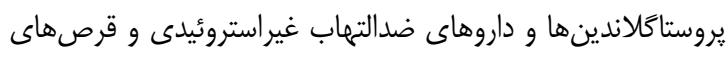

دوره آr، شماره r.، ياييز

فصلنامه افق دانش 


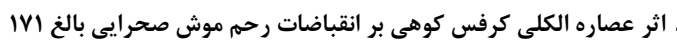

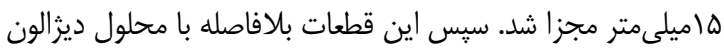
شامل؛

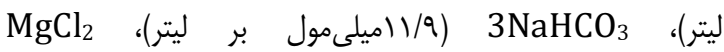

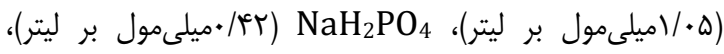
( NaCl

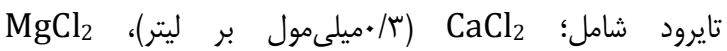

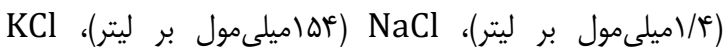
(ه/ه/هيلىمول بر ليتر)، (هaHCO

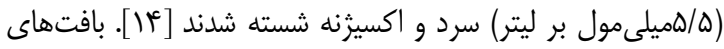

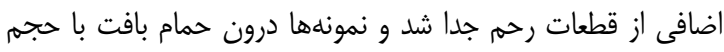

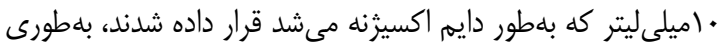
كه از يك طرف به كيره استيل در ته حمام بافت و از باز بالا بهوسيله

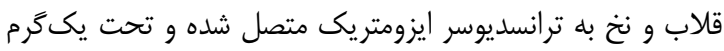

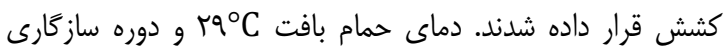

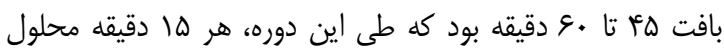

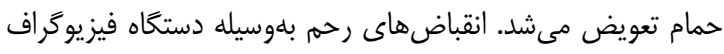
Harvard Universal Oscillograph) / / ميلىمتر در ثانيه روى كاغذ ثبت شد. بلمنظور جلوكيرى از تغيير

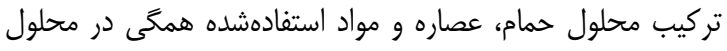
تايرود يا ديزالون حل شدند.

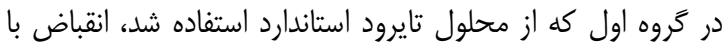

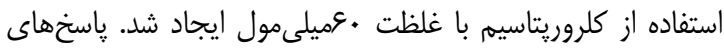

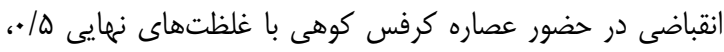

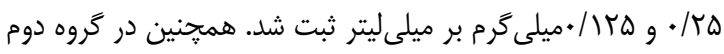

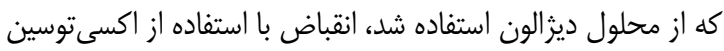
إباد (Weimer Pharma)

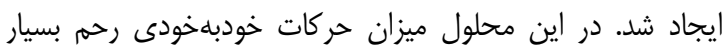

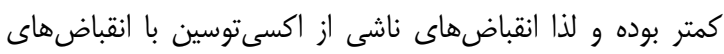

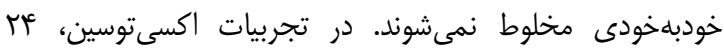

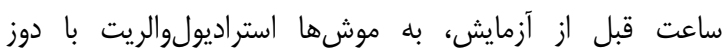

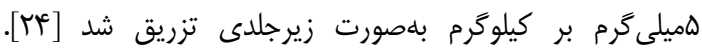

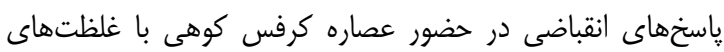

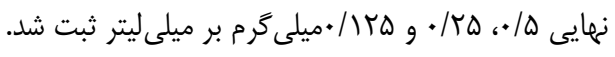

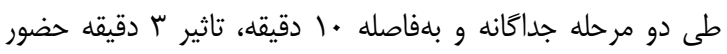

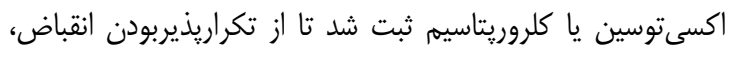

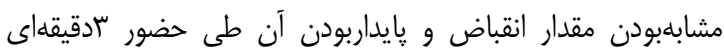

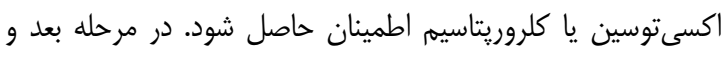

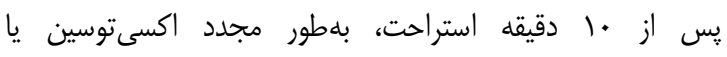

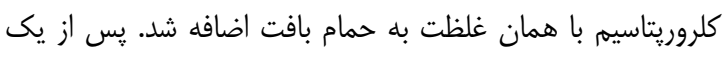

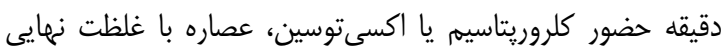

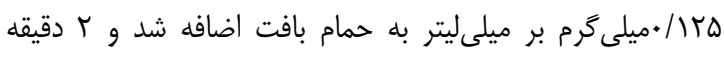

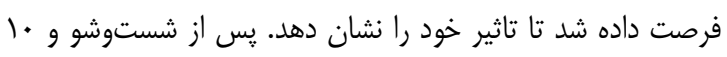

كرد [19]. براساس مطالعه صورتگَرفته، عصاره كرفس مىتواند شدت اثر و طول دوره اثر فنوباربيتول و آمينوييرين را اف افزايش دهارئه

علىرغم مطالبى كه در بالا اشاره شد، در بررسى منابع اطلاعرسانى

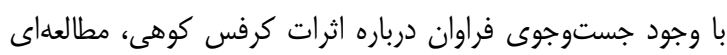

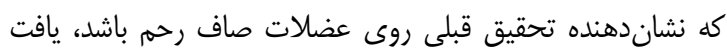

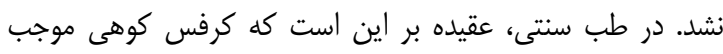

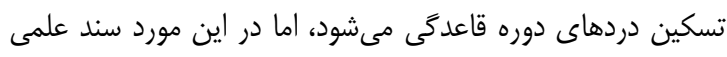

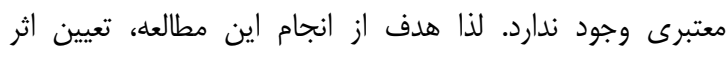

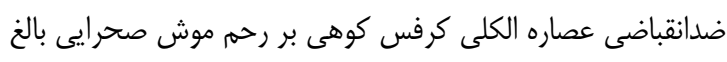

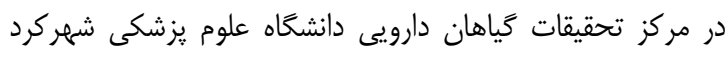

بود.

\section{مواد و روشها}

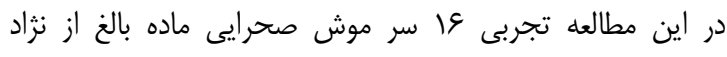

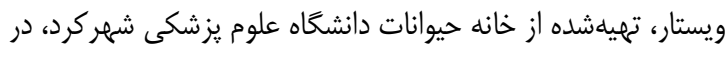

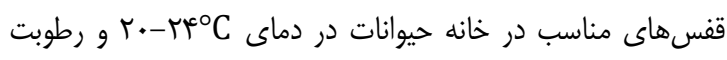

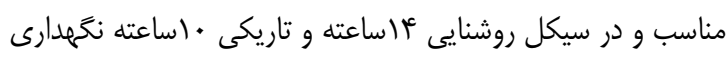

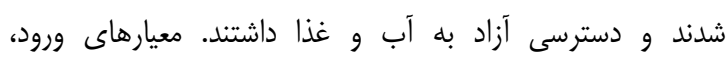

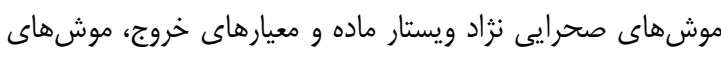

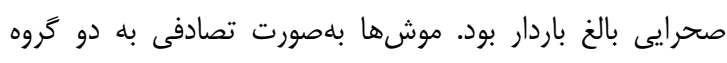

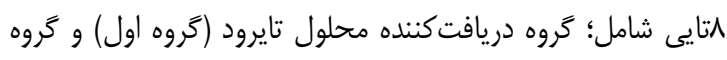

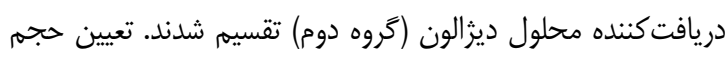
نمونه براساس مقالههاى مشابه بود.

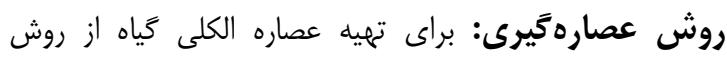

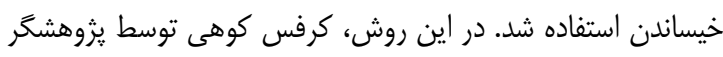

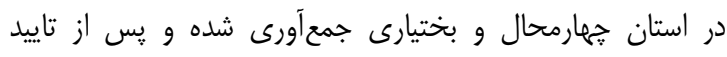

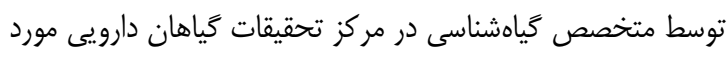

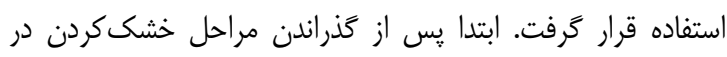

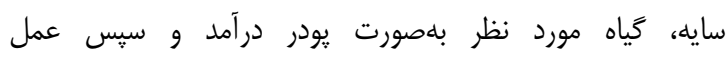

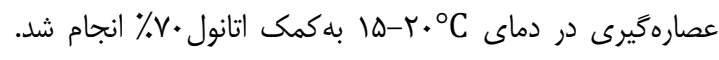

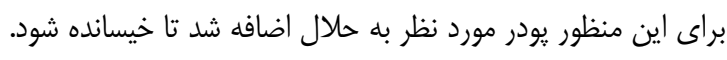

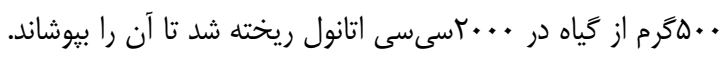

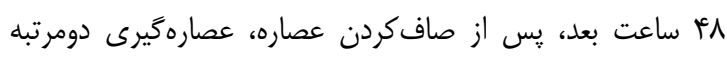

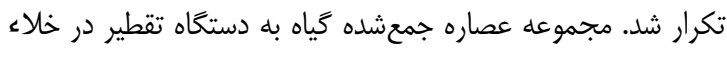

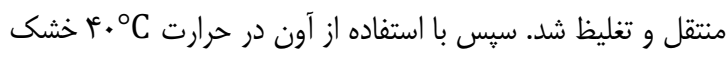

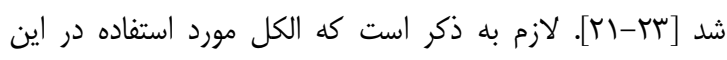

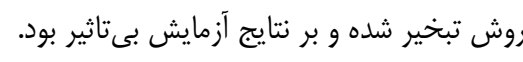

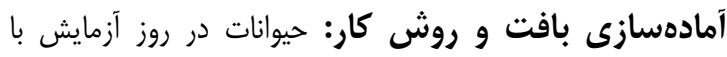

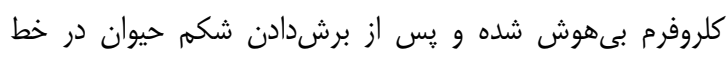

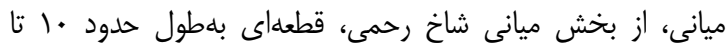


L L آن در رحم بهاثبات رسيده است [عَ] را باز نموده و با افزايش

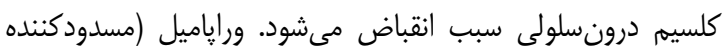

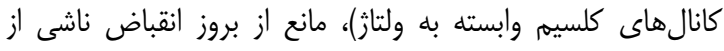

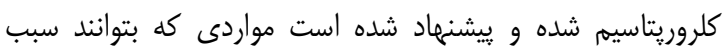

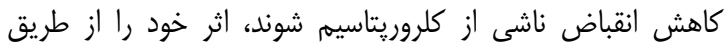

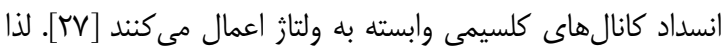
بلنظر مىرسد بخشى از عملكرد مهارى عصاره كرفس كوهى با مالئ همين روش انجام شده باشد.

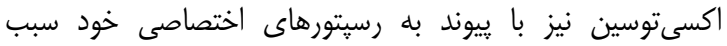

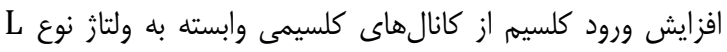

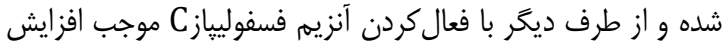

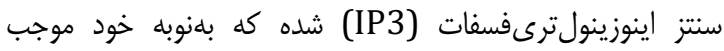

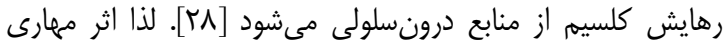

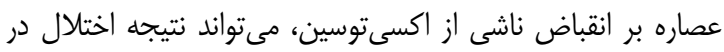

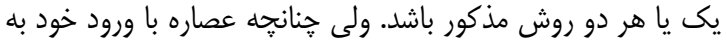

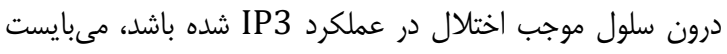

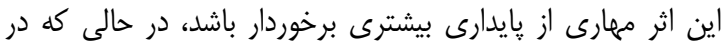

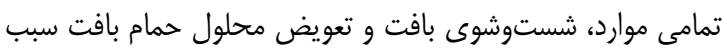

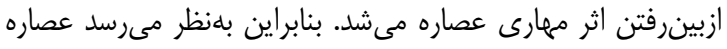

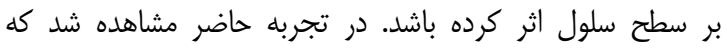

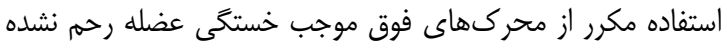

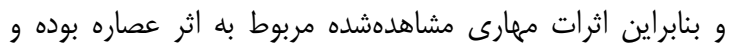
نتيجه خستخى بافت نبوده است. مطالعات زيادى اثرات Z-ليخوستيليد را مورد بررسى قرار دادهاند. بلعنوان نمونه مشخص شده است كه اين ماده سبب شلش الشدان

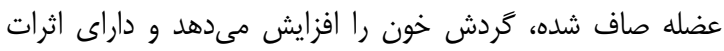

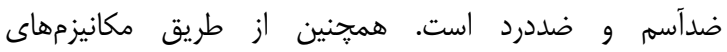

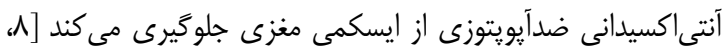

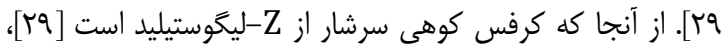

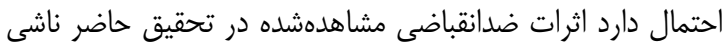
از ماده Z-ليكوستيليد موجود در عصاره كرفس كوهى بات باشد.

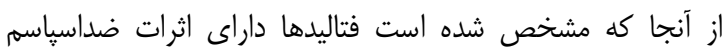

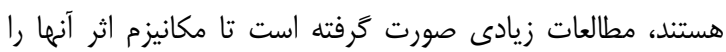

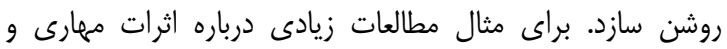

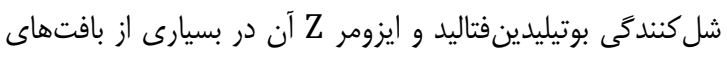
بدن مثل عروق مختلف خونى، روده، دستخاه تنفس و دوستيات

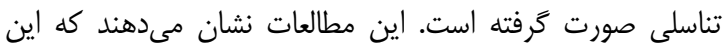

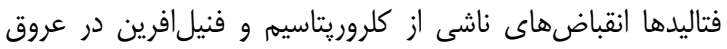

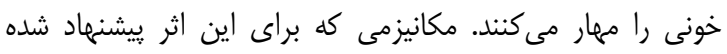

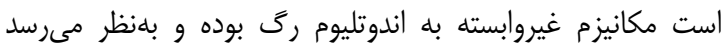

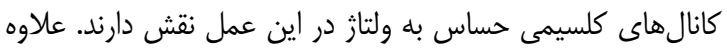
بر تاثير كانالهاى كلسيمى حساس به ولتاثر، مكانيزم ديخرى كه

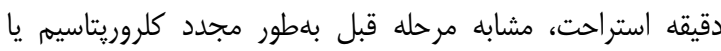

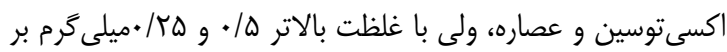

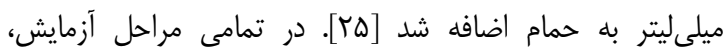

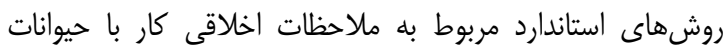
رعايت شد و موشها در ابتدا در شرايط استاندارد بلوسيله كلروفرم بى هوش شده و پِ از خارجنمودن شاخ رحمى كشته شدند.

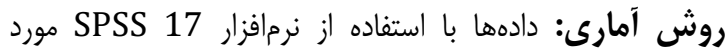

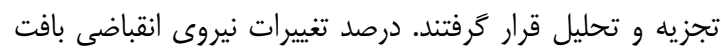

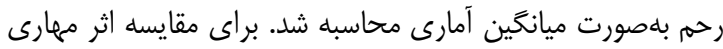

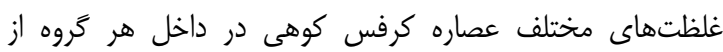
آزمون آناليز واريانس بلروش انداف عارهار كرفاى تكرارى استفاده شد.

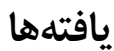

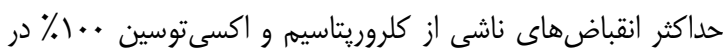

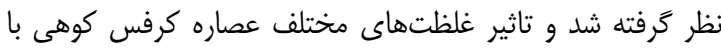
آن سنجيده شد. عصاره كرفس كوهى بلهور معنىدار بلهورت وابسته به غلظت سبب كاهش نيروى انقباضى رحم موش برون صحرايى ناشى از

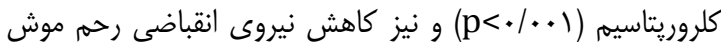

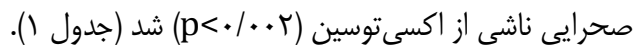

جدول () مقايسه ميانكين آمارى درصد اثر مهارى غلظتهاى مختلف عصاره

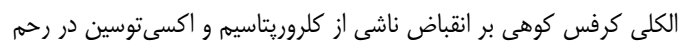

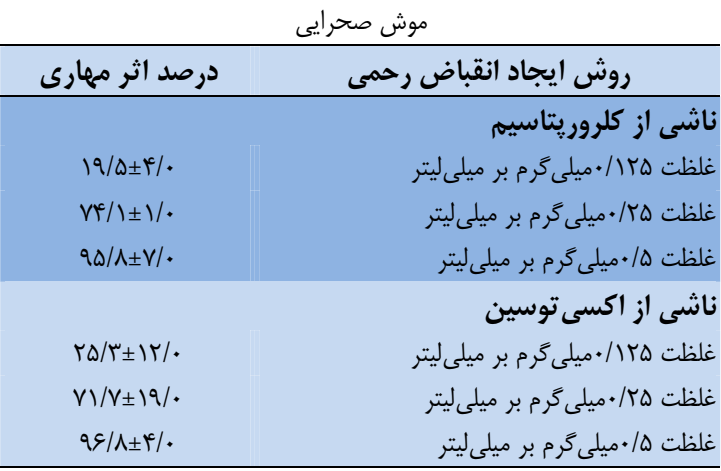

بحث

با توجه به نتايج اين مطالعه، عصاره الكلى برك كرفس كوهى

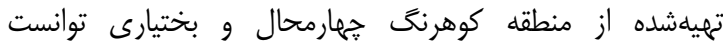

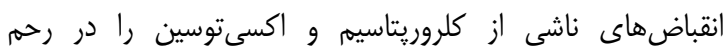
موشهاى صحرايى كاهش دهد. كاهش انقباضهاى رحمى در هر إنى

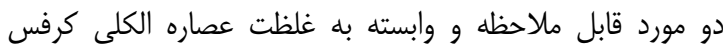

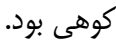

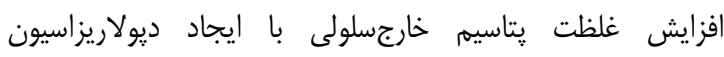
سلولهاى عضله صاف، كانالهاى كلسيمى وابسته به ولتاز كه نوع 


\section{نتيجه كيرى}

عصاره الكلى كرفس كوهى، اثر مهارى وابسته به غلظت بر

انقباضهاى رحمى موش صحرايى نزاد ويستار ناشى از كلروريتاسيم

و اكسىتوسين دارد.

تشكر و قدردانى: اين تحقيق حاصل پايان نامه يزشكى عمومى

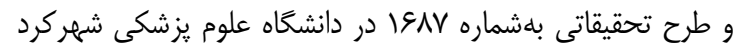

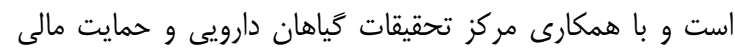

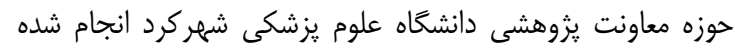

است كه بدين وسيله از زحماتشان تقدير و تشكر معى شود.

تاييديه اخلاقى: در تمامى مراحل آزمايش، روشهاى استاندارد مربوط به ملاحظات اخلاقى كار با حيوانات رعايت شد.

تعارض منافع: موردى از طرف نويسندكان بيان نشده است.

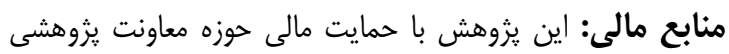

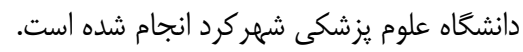

منابع

1- French L. Dysmenorrhea in adolescents: Diagnosis and treatment . Pediatr Drugs. 2008;10(1):1-7.

2- Serashti M, Azari P, Rafiean M, Kheiri S. Use of herbal medicines by pregnant women in Shahr-e-Kord. J Reprod Infertility. 2006;7(2):125-31.

3- Omidbaigi R, Sefidkon F, Saeedi K. Essential oil content and composition of Kelussia odoratissima Mozaff as an Iranian endemic plant. J Essent Oil Bearing Plants. 2008;11(6): 594-7.

4- Salimi M, Ebrahimi A, Shojaee Asadieh Z, Saei Dehkordi SS. Essential oil composition of Kelussia odoratissima Mozaff. Ir J Med Aromatic Plants. 2010;26(2):147-56.

5- Yousefzadeh Z, Karimi A. Anti-inflammatory Effects of Kelussia odoratissima in Rats Model of Rheumatoid Arthritis. Am J Ethnomed. 2014;1(1):50-5.

6- Askari-Khorasgani 0, Mortazaeinezhad F. Chemical composition of wild celery (Kelussia odoratissima) cultured in-vitro and from its local habitat. J Essent Oil Bear Pl. 2015;18(2):489-95.

7- Asgary S, Naderi G, Dashti G, Paknahad Z. Effect of Amirkabiria odorastissima mozaffarian on the development and progression of fatty streaks in hypercholesterolemic rabbits. Phytother Res. 2004;18(5):370-2.

8- Rabbani M, Sajjadi SE, Sadeghi M. Chemical composition of the essential oil from Kelussia odoratissima Mozaff and the evaluation of its sedative and anxiolytic effects in mice. Clinics (Sao Paulo) 2011;66(5):843-8.

9- Sajjadi SE, Shokoohinia Y, Mehramiri P. Isolation and characterization of steroids, phthalide and essential oil of the fruits of Kelussia odoratissima Mozaff., an endemic mountain celery. Res Pharm Sci. 2013;8(1):35-41.

10- Kheirandish M, Behbahani M. Evalution of cytotoxic effect of leaves, shoots, seeds, flowers and roots extracts of Kelussia odoratissima Mozaff grown in Kohrang and Freydonshahr regions. Res Med. 2014;38(4):221-5. [Persian]
براى اثر E-ليخوستيليد در سيستم عصبى مركزى بيشنهاد شده

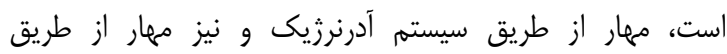

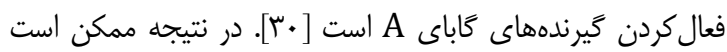

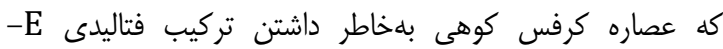

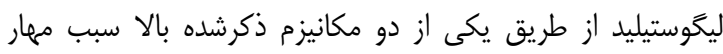
انقباض هاى رحمى شود. در مطالعات ديخر مشخص شده است كه فتاليدهاى موجود درد بردى

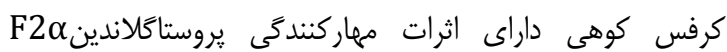

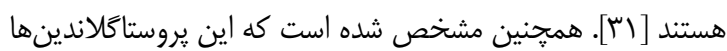

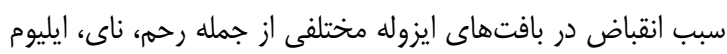

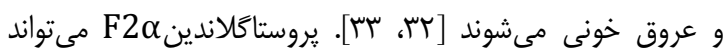

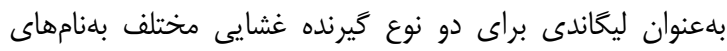

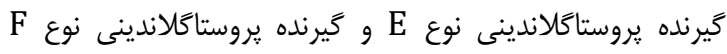

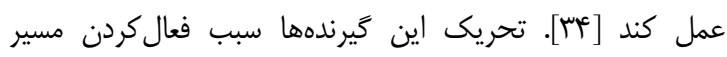

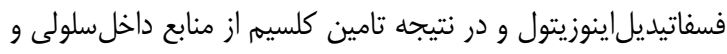

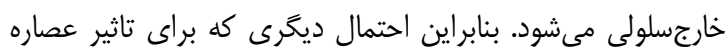

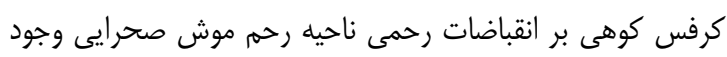

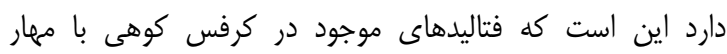

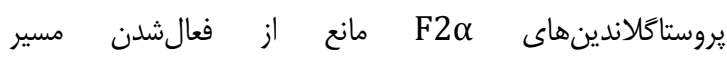

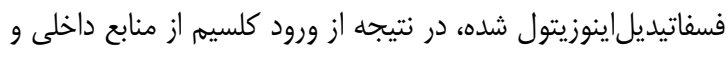

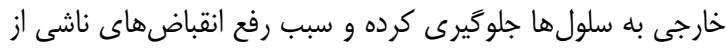

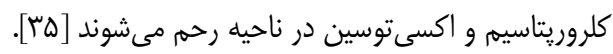

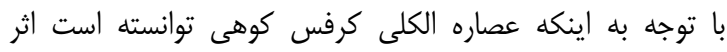

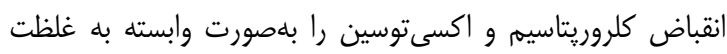

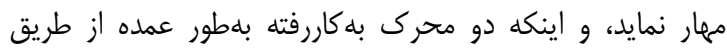

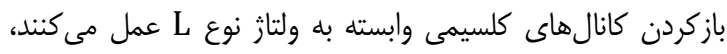

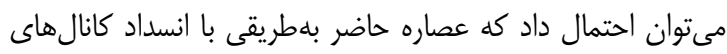

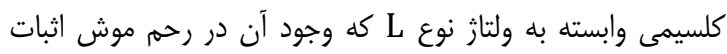

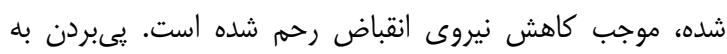

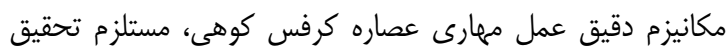

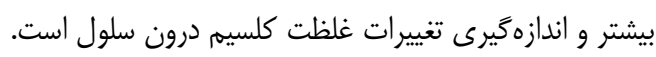

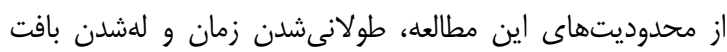

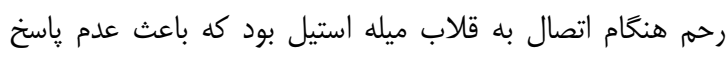
بافت در بعضى از موارد به تحريكات شد كه با با استفاده از قالاب داب

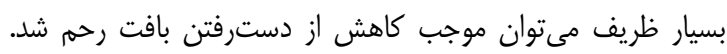

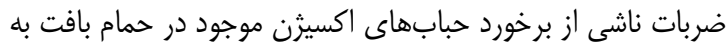

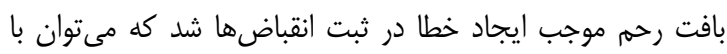

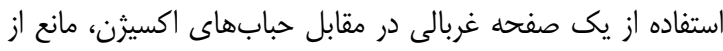

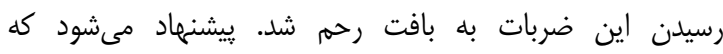

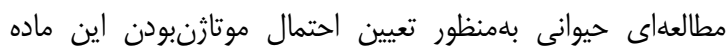

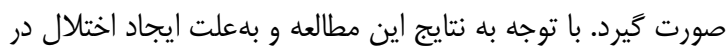

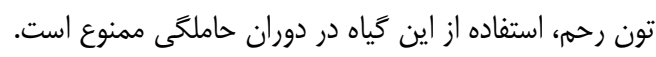


induced seizures in male mice. J Med Plants. 2012;11(4):193-201. [Persian]

24- Gharib Naseri MK, Mard SA, Farbood Y. Effect of Anethum Graveolens fruit extract on rat uterus contractions. Iran J Basic Med Sci. 2006;8(4):263-70. [Persian]

25-Gharib Naser MK, Mazlumi H, Goshayesh M, Vakilzade G, Heydari A. Anhispasmodic effect of Zataria multiflorn Boiss. Leuf Extract on the rat uterus. Ir J Pharmaceutical Res. 2006;5(2):131-36.

26- Ohya Y, Sperelakis N. Fast $\mathrm{Na}+$ and slow $\mathrm{Ca}^{2+}$ channels in single uterine muscle cells from pregnant rats. Am J Physiol. 1989;257(2 Pt 1):C408-12.

27- Kim BK, Ozaki H, Lee SM, Karaki H. Increased sensitivity of rat myometrium to the increased contractile effect of platelet activating factor before delivery. Br J Pharmacol. 1995;115(7):1211-4.

28- Sanborn B.M. Hormones and calcium: Mechanisms controlling uterine smooth muscle contractile activity. The Litchfield Lecture. Exp Physiol. 2001;86(2):223-37.

29- Asadiyeh Shojaei Z, Ebrahimi A, Salimi M. Chemical composition of three ecotypes of wild celery (Kelussia odoratissima). J Herb Spice Med Pl. 2011;17(1):62-8.

30- Bunel V, Antoine MH, Nortier J, Duez P, Stévigny C. Nephroprotective effects of ferulic acid, Z-ligustilide and E-ligustilide isolated from Angelica sinensis against cisplatin toxicity in vitro. Toxicol In Vitro. 2015;29(3):458-67.

31- Salimi M, Ebrahimi A, Saei-Dehkordi S S, Shojaei Z. Extraction and Identification of Chemical Composition of Klussia odoritissima Mozaff. Iran J Med Aromatic Plants. 2010;26(2):147-56. [Persian]

32- Phillippe $M$, Saunders T, Basa A. Intracellular mechanisms underlying prostaglandin F2 $\alpha$-stimulated phasic myometrial contractions. Am Physiol Society. 1997;273(4):E665-73.

33- Beretta C, Cavalli M. A sheer pharmacologic approach to compare the contractile effects of PGF2 $\alpha$, DLcloprostenol and D-cloprostenol on isolated uterine, tracheal, ileal and arterial smooth muscle preparations. Theriogenology. 2004;62(5):837-46.

34- Narumiya S, Sugimoto Y, Ushikubi F. Prostanoid receptors: structures, properties and functions. Physiol Rev. 1999;79(4):1193-226.

35- Gharib Naseri MK. Inhibitory effect of Zizyphus Spina Christi leaf extract on rat isolated uterus. Jundishapur Sci Med J. 2003;38(2):24-31. [Persian]
11- Cao YX, Zhang W, He JY, He LC, Xu CB. Ligustilide induces vasodilatation via inhibiting voltage dependent calcium channel and receptor-mediated $\mathrm{Ca} 2+$ influx and release. Vascul Pharmacol. 2006;45(3):171-6.

12- Kerry N, Rice-Evans C. Peroxynitrite oxidises catechols to o-quinones. FEBS let. 1998;437(3):167-71.

13- Lee TF, Lin YL, Huang YT. Studies on antiproliferative effects of phthalides from Ligusticum chuanxiong in hepatic stellate cells. Planta Med. 2007;73(6):527-34.

14- Ahmadi F, Kadivar M, Shahedi M. Antioxidant activity of Kelussia odoratissima Mozaff. in model and food systems. Food Chem. 2007;105(1):57-64.

15- Iravani M, Jaberol-Ansar Z. Kelussia odoratissima, an overthrowing plant in Central Zagros region. Tehran: Payam Sabz Publ. 2005. p. 39.

16- Asgari S, Naderi Gh, Jafariyan A, Askari N, Behagh AR. Fibrinolytic activity of Amirkabiria Odoratissima Mozaffarian. J Med plants. 2005;1(13): 19-25.

17- Asgary S, Naderi Gh, Dashti Gh, Paknahad Z. Effect of Amirkabiria odoratissima Mozaffarian on development and progression of fatty streaks in hypercholesterolemic rabbits. Phytotherapy Res. 2004;18(5):370-2.

18- Ko FN, Huang TF, Teng CM. Vasodilatory action mechanisms of apigenin isolated from Apium graveolens in rat thoracic aorta. Biochim Biophys Acta. 1991;1115(1):69-74.

19- Hojati MR, Sedighi Hafshejani M, Shahrani M. Effect of alcoholic extract of Kelussia odoratissima Mozaffarian on ileum contractions in rats. J Sabzevar Uni Med Sci. 2012;19(2):156-63.

20- Jakovljevic V, Raskovic A, Popovic M, Sabo J. The effect of celery and parsley juices on pharmacodynamics activity of drugs involving cytochrome P450 in their metabolism. Eur J Drug Metab Pharmacokinet 2002;27(3):153-6.

21- Mehrabani M, Mehrabani M, Nematolahi $H$, Mehrabani M, Mansourinejad E, Raftari Sh. Effects of apple (Malus domestica Borkh.) diet on rat reproduction and sex ratio of offsprings. J Kerman Univ Med Sci. 2011;18(3):260-70.

22- Duraipandiyan V, Ayyanar M, Ignacimuthu S. Antimicrobial activity of some ethno medicinal plants used by Player tribe from Tamil Nadu. BMC Complement Altern Med. 2006;6:35.

23- Naderi F, Azhdari-Zarmehri H, Erami E, Sonboli A, Sofiabadi M, Mohammad-Zadeh $M$. The effect of Tanacetum sonbolii hydroalcholic extract on PTZ 